Recepción: 09 de abril de 2017

Aceptación: 09 de junio de 2017

Publicación: 14 de junio de 2017

\title{
DISEÑO Y CONSTRUCCIÓN DE UN EQUIPO PELADOR DE MAÍZ DE BAJO COSTO PARA LA VARIEDAD SECO SUAVE
} DE ALTURA

\section{DESIGN AND CONSTRUCTION OF A LOW PRICE CORN PEELING MACHINE FOR SOFT DRY VARIETY OF HEIGHT}

Melton E. Tapia Zurita ${ }^{1}$

Edison Tapia Zurita²

Sandra Arla Odio ${ }^{3}$

1. Ing. Mecánico, Máster en Mecatrónica por la Universidad Politécnica de Cataluña, Docente, Coordinador del área Mecatrónica del Departamento de Ciencias de la Energía y Mecánica de la Universidad de Fuerzas Armadas - ESPE, Provincia de Pichincha, Ecuador. E-mail: metapia@espe.edu.ec

2. Ing. Mecánico, Máster en Materiales, Diseño y Automatización por la Escuela Politécnica Nacional de Quito, jefe de proyectos SEDEMI (Soluciones en Estructuras Metálicas), provincia de Pichincha, Ecuador.E-mail: gustavo.zur@hotmail.com

3. Ing. Mecánico y Máster en Medio Ambiente por la Universidad de La Habana, Cuba, Docente del Departamento de Ciencias de la Energía y Mecánica de la Universidad de Fuerzas Armadas -ESPE, Provincia de Pichincha, Ecuador. E-mail: smarla@espe.edu.ec

\section{Citación sugerida:}

Tapia Zurita, M.E., Tapia Zurita, E. y Arla Odio, S. (2017). Diseño y construcción de un equipo pelador de maíz de bajo costo para la variedad seco suave de altura. 3C Tecnología: glosas de innovación aplicadas a la pyme, 6(2), 52-64. DOI: <http://dx.doi.org/10.17993/3ctecno.2017.v6n2e22.52-64/>. 


\section{RESUMEN}

El trabajo determina los parámetros generales de diseño y montaje de elementos destinados a realizar el proceso de pelado (deshojado) de maíz de la variedad seco suave cultivado en la región andina de Sudamérica. Las relaciones dimensionales y parámetros determinados, pueden ser aplicados en el desarrollo de máquinas de distintas capacidades y dimensiones así como para procesos de trabajo similares, todas ellas con costos de fabricación inferiores a los encontrados en el mercado; de allí el aporte del estudio. Por la naturaleza del proceso y la gran cantidad de variables presentes, condición que dificulta la determinación netamente teórica de los parámetros de trabajo, se utiliza la metodología de diseño de experimentos para la obtención de los mismos. Para la realización de pruebas que permitan obtener información de factores como formas, materiales, velocidades de trabajo, separación de elementos y arquitectura en general, se utilizan prototipos enfocados de experimentación que entregan información para la consecución del objetivo planteado.

\section{ABSTRACT}

The work determines the general parameters of design and assembly of the elements destined to carry out the peeling process of maize of the soft dry variety cultivated in the Andean region of South America. The dimensional relationships and determined parameters can be applied in the development of machines of different capacities and dimensions, as well as for similar work processes, all of them with lower manufacturing prices than those found in the commerce; hence the contribution of the study. Due to the nature of the process and the large number of variables present, a condition that makes the purely theoretical determination of the work parameters difficult, the methodology of experiment design is used to obtain them. To carry out tests that allow obtaining information of factors such as shapes, materials, work speeds, element separation and architecture in general, we use focused experimental prototypes that deliver information to achieve the stated objective.

\section{PALABRAS CLAVE}

Parámetros, diseño, deshojado, maíz, Sudamérica.

\section{KEY WORDS}

Parameters, design, leafless, corn, South América. 


\section{INTRODUCCIÓN}

La Región Andina Sudamericana produce una variedad de productos agrícolas que son dedicados al consumo interno y a la exportación, y uno de estos productos es el maíz suave de altura. La agricultura familiar es la predominante en América Latina y el Caribe con un $70 \%$ de las unidades productivas agrícolas; pero, a pesar de la gran cantidad de territorio que posee, es la que menores rendimientos presenta respecto a la agricultura comercial (CEPAL, FAO, IICA, 2014). Esta problemática es debida a la falta de tecnificación y modernización en los procesos productivos (De los Ríos, 2008), a diferencia de lo que se hace en los países tecnológicamente desarrollados en los que la tecnificación ha permitido mayor producción y competitividad.

En el Ecuador, particularmente y como ejemplo del problema existente en el sector agrícola, se conoce que el $93 \%$ de productores no tiene acceso a créditos ni a asistencia técnica, tampoco tienen educación ni formación alguna en un 88\%; y, mientras en la Unión Europea el 90\% de productores agrícolas utiliza maquinaria agrícola como tractores, en el Ecuador, por ejemplo, únicamente lo hace un 1\% (Inurritegui, M., Camacho, I., Ludeña, J., 2008).

En lo referente a maquinaria agrícola, todos los países de América Latina, excepto Brasil, son importadores netos de la misma (Donoso, 2007). Las grandes Corporaciones abastecen de maquinaria a la región con plantas ubicadas en México y Brasil, existiendo un gran número de fabricantes locales que aprovechan oportunidades en espacios de mercado no cubiertos por las grandes corporaciones (Donoso, 2007). Esto se debe, posiblemente, a que estos espacios no representan un mercado económicamente atractivo y es a este espacio a donde se destinó el presente estudio.

En el mercado latinoamericano, especialmente en países de la región Andina, la oferta de maquinaria agrícola para aplicaciones específicas es nula y se trata de adaptar la maquinaria existente para el procesamiento de variedades propias de la región que no han sido estudiadas, en la mayor parte de casos de manera infructuosa debido a las características específicas de dichos productos, a los elevados precios del equipamiento y a la falta de conocimiento por parte de los productores.

Estudios similares al propuesto se conoce que fueron desarrollados y aplicados en las décadas de 1940 y 1960 en Estados Unidos, pero presentaron inconvenientes con la cantidad de maíz desgranado en el proceso y daños en las hojas del maíz (Cruz, P., Romantchick, E., Hahn, F., 2010). En el año 1970 se patentó un dispositivo compuesto por rodillos paralelos que giran en sentidos opuestos y que se componen de una sucesión de piezas metálicas y plásticas apiladas sobre el eje (España Patente no $384194,1970)$.

En el año 2010, en la Universidad de Chapingo, México, se desarrolla un prototipo de deshojado de maíz para la obtención de hojas para tamales, el mismo que utiliza rodillos lisos que giran en sentidos opuestos utilizando un motor de 1,5 HP (Cruz, P., Romantchick, E., Hahn, F., 2010). 
En la Universidad de Fuerzas Armadas - ESPE, en el año 2014, se desarrolló el proyecto de tesis de pregrado denominado "Diseño y construcción de una máquina deshojadora de maíz seco que partiendo de la mazorca con su envoltura, entregará como producto la mazorca y como subproducto, la envoltura del maíz" (Moya, 2014) que fue desarrollado con la finalidad de experimentar con un prototipo de rodillos lisos y transmisión de potencia mediante engranajes además de corroborar información referente a dimensiones de la variedad de maíz en estudio y determinar las fuerzas que intervienen en el trabajo.

En el gráfico 1 se pueden observar rodillos comerciales destinados al proceso de pelado de maíz de variedad seco duro que es la de mayor producción en el mundo. No se ha encontrado información alguna de parámetros de diseño de estos equipos como materiales, durezas, montaje, ensayos, etc. Se obtienen únicamente especificaciones técnicas básicas que poco o nada aportan en el desarrollo de sistemas adaptados a otras variedades.

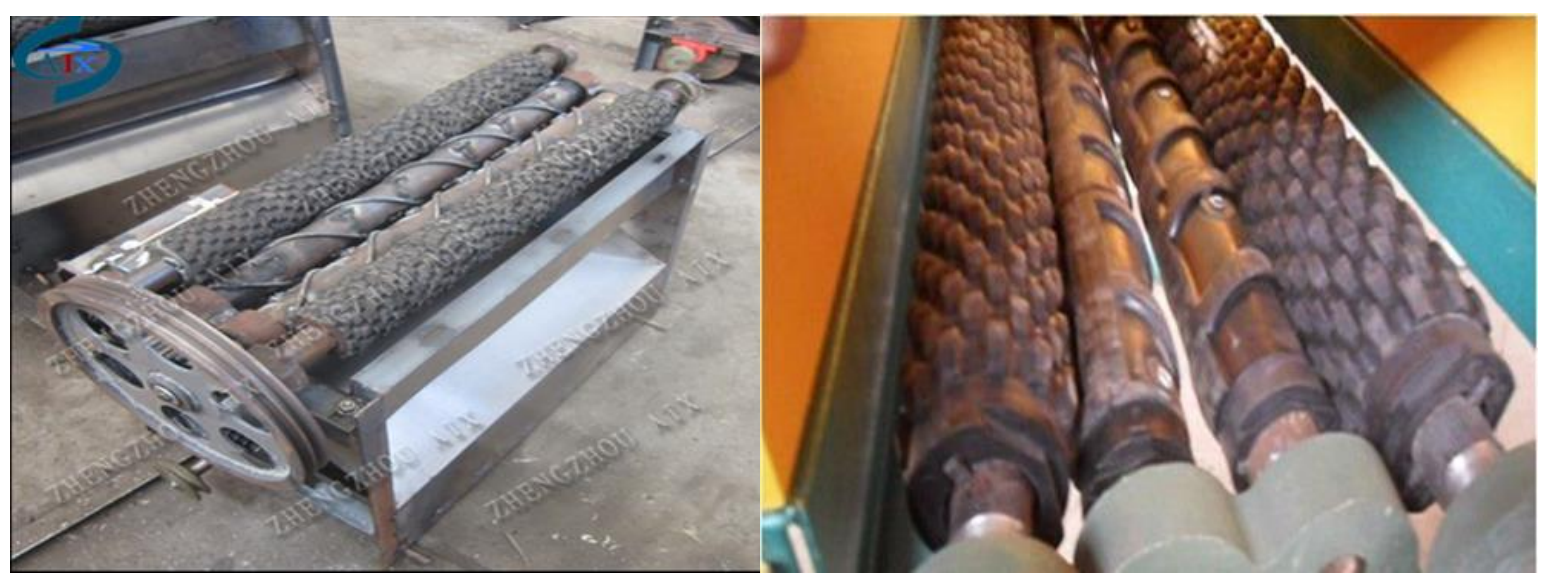

Gráfico 1. Rodillos peladores comerciales para variedad seco duro.

Fuente: Internet.

Este trabajo muestra los resultados obtenidos para el establecimiento de la arquitectura del equipo pelador, así como detalles de los materiales, formas y condiciones de montaje de los elementos, de manera que el trabajo se realice de manera efectiva y eficiente.

En el procesamiento de otras variedades del producto, las relaciones entre parámetros de trabajo deben ser analizadas particularmente dependiendo de varios factores como: tamaño, forma, relaciones dimensionales y factores de ruido que puedan existir, pero pueden seguir los lineamientos encontrados en los resultados del presente artículo. 


\section{METODOLOGÍA}

Con la finalidad de minimizar los riesgos técnicos y de mercado, con resultados de desarrollo que estén acordes a las necesidades de los potenciales usuarios, se utiliza la metodología de diseño y desarrollo de productos (Ulrich, K., Eppinger, S., 2013), poniendo énfasis en el proceso de desarrollo de conceptos que incluye la realización de las siguientes actividades:

- Identificar necesidades de los usuarios

- Establecer especificaciones objetivo

- Desarrollar conceptos

- Seleccionar conceptos

- Probar conceptos

- Establecer especificaciones finales

- Realizar el desarrollo descendente

Este proceso permite delinear durante todo el proceso de creación del producto los parámetros de trabajo y validarlos técnica y funcionalmente través de pruebas de operación y retroalimentación de usuarios para llegar finalmente a un producto que satisfaga los requerimientos técnicos y de mercado.

El método sugiere desarrollar durante todo el proceso de desarrollo, distintos tipos de prototipos que pueden ser físicos, analíticos, integrales o enfocados, que permitan despejar dudas respecto a funcionamiento, integración, alcance de objetivos planteados y/o comunicación del proyecto.

Como parte fundamental del método, se encuentra el estudio minucioso de máquinas similares y sus costos, ello para determinar de forma real las innovaciones y/o mejoras en aspectos técnicos, económicos y de producción que presentará el estudio.

\section{RESULTADOS}

En concordancia con la metodología empleada, fueron desarrollados una serie de conceptos de los cuales varios de ellos fueron implementados para la realización de pruebas operacionales a través de prototipos enfocados, siendo el prototipo mostrado en el gráfico 2 el que demostró adaptarse a las necesidades de los usuarios en la mayor parte de sus requerimientos y el que técnicamente presentó mejor desempeño operacional. 


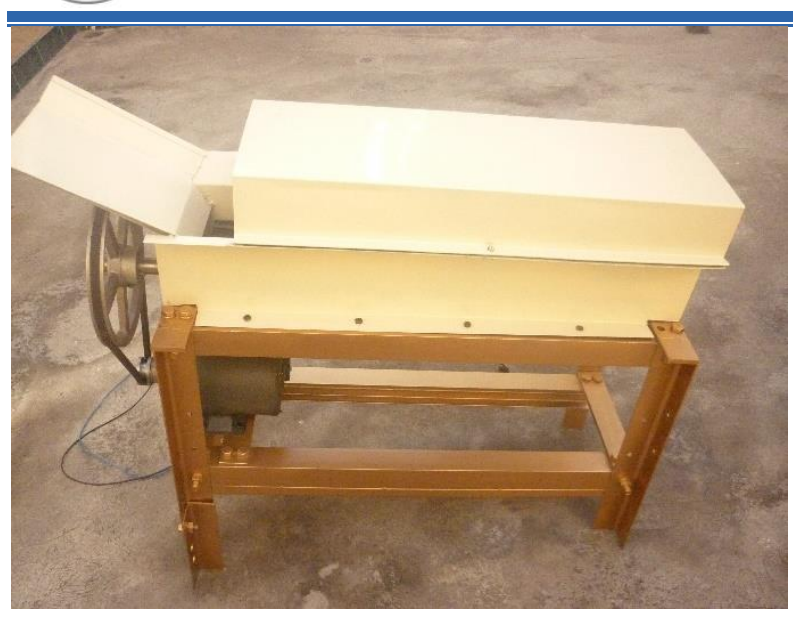

Gráfico 2. Equipo pelador doble rodillo. Fuente: elaboración propia.

El diseño tiene una arquitectura modular tipo ranura para que su montaje y desmontaje se realice fácilmente. Para comodidad en su producción y/o estandarización de partes y componentes, todos los trozos funcionales y físicos están compuestos por varios elementos que se acoplan y desacoplan con facilidad.

Los sistemas funcionales del equipo son los siguientes:
a.- Alimentación y guiado
b.- Pelado
c.- Soporte
d.- Eléctrico

\section{SISTEMA DE ALIMENTACIÓN Y GUIADO}

Este sistema tiene la función de permitir el ingreso del producto de forma fácil y segura al proceso de pelado e impedir que el material, o parte de éste, salga del área de trabajo; está fabricado en lámmina de acero A 36 con espesor de $1 \mathrm{~mm}$ y el ángulo de inclinación de la rampa de acceso es de $35^{\circ}$, el mismo que de acuerdo a las pruebas realizadas permite que el material que ingresa al proceso tenga el posicionamiento correcto y logre de esta manera el contacto del producto con las caras de fricción de los elementos de trabajo. La utilización de distintos ángulos provocó atascamientos de la mazorca entre los rodillos, en unos casos, y en otros, que la misma salga sin procesarse completamente.

\section{SISTEMA DE PELADO}

Se compone de dos rodillos de caucho que giran en direcciones opuestas y llevan la mazorca al área de unión de los dos rodillos, direccionado la misma y a la vez generando el rozamiento necesario para desprender la hoja. Los rodillos tienen un diámetro exterior de $85 \mathrm{~mm}$. sobre el cual se encuentran 
mecanizados 10 dientes de $11 \mathrm{~mm}$. de profundidad, $10 \mathrm{~mm}$. de ancho en la circunferencia exterior y $14 \mathrm{~mm}$. en la circunferencia de base. La longitud de los rodillos en el área útil de proceso es de 700 $\mathrm{mm}$. Con este diseño se reduce el costo de manufactura de los rodillos y se disminuye la longitud de los mismos, ahorrando costos en materiales y manufactura.

Los dientes de los rodillos tienen un ángulo de $30^{\circ}$ respecto al eje axial.

El caucho tiene una dureza shore A 75.

En el gráfico 3 se muestran los rodillos fabricados para la función de pelado.

Con la finalidad de que los rodillos cumplan la función y no realicen mordedura de la mazorca es necesario que los mismos mantengan una determinada distancia entre centros. Esta separacion tiene un valor que está en función del diámetro del rodillo, el diámetro de la mazorca y el ángulo de deslizamiento. Otra innovación de este equipo es la posibilidad de deslizar los rodillos dependiendo de la variedad de producto con la que se trabaje, condición que no poseen los equipos comerciales.

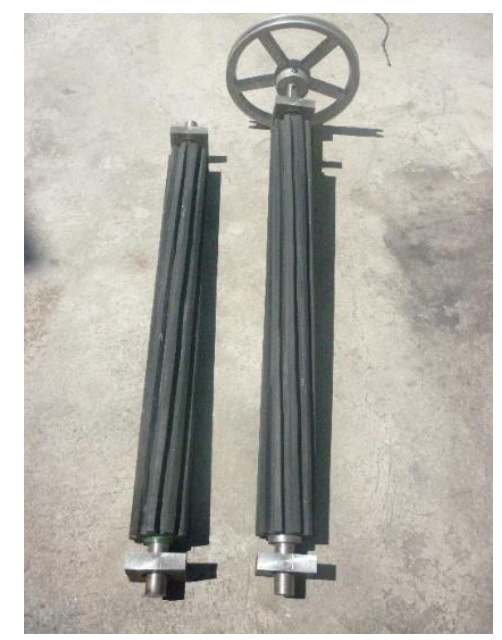

Gráfico 3. Rodillos peladores.

Fuente: elaboración propia.

El gráfico 4 muestra la medida de dureza del caucho de los rodillos.

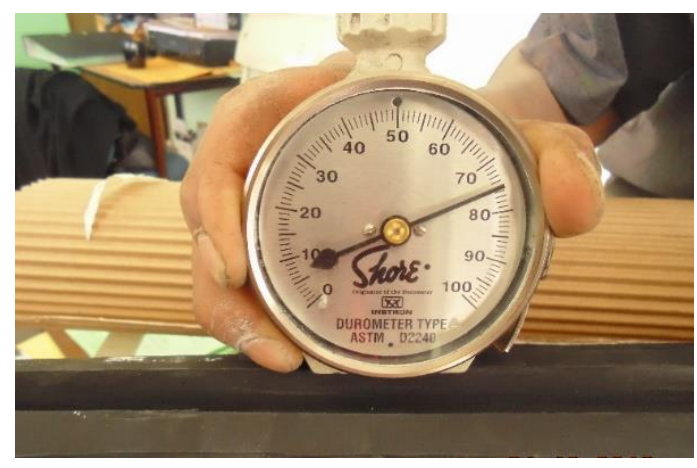

Gráfico 4. Medida de dureza de los rodillos. Fuente: elaboración propia. 
El ángulo de deslizamiento de la mazorca $(\theta)$, está determinado por la fuerza normal que ejerce el rodillo sobre la misma y la fuerza resultante. El gráfico 5 muestra las relaciones dimensionales entre los rodillos y la mazorca en el momento del proceso.

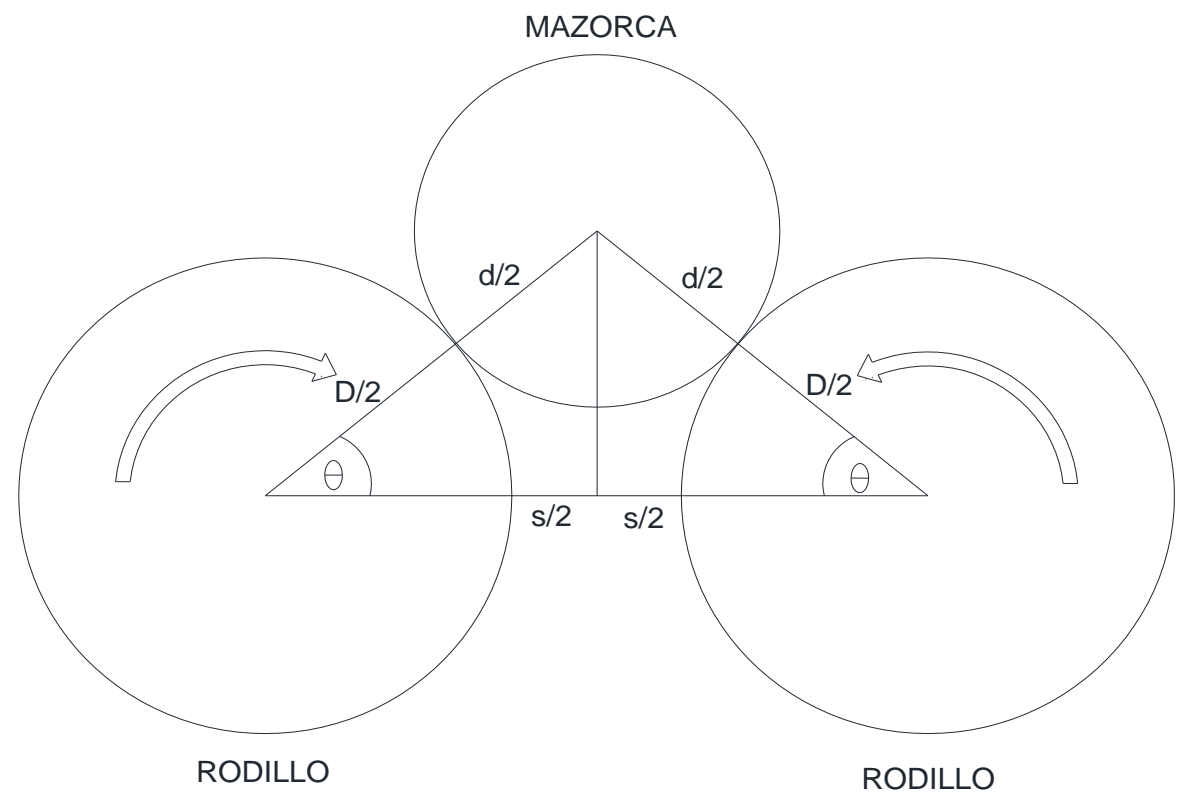

Gráfico 5. Relaciones dimensionales entre rodillos y mazorca.

Fuente: elaboración propia.

En base a lo mostrado en el gráfico 5, se puede determinar la distancia exacta que debe existir entre los ejes de los rodillos para que el proceso se realice con la máxima eficiencia. Esta condición se logra teniendo la dirección de la fuerza resultante paralela a la dirección del eje de unión de los centros de los rodillos.

La distancia que debe existir entre los centros de los rodillos la obtenemos de la siguiente manera:

$$
\begin{aligned}
& (D / 2+d / 2) \cos \theta=D / 2+s / 2 \\
& 2((D / 2+d / 2) \cos \theta-D / 2)=s
\end{aligned}
$$

La distancia entre los centros de los ejes es:

$$
D C=2 D / 2+s
$$

$$
D c=D+2((D / 2+d / 2) \cos \theta-D / 2)
$$

Por tanto obtenemos que:

$$
D c=2\left(\frac{D}{2}+\frac{d}{2}\right) \cos \theta
$$


Si la distancia entre centros es mayor a la calculada, la fuerza resultante se dirigiría hacia abajo, por tanto la mazorca tendería a quedar atrapada entre los rodillos. En el caso opuesto, es decir si la distancia es menor a la calculada, la fuerza resultante se dirigiría hacia arriba, esto ocasionaría que el producto sea empujado hacia arriba y no exista el contacto suficiente para que el proceso se realice de manera eficiente.

\section{SISTEMA DE SOPORTE}

Este sistema cumple doble función, la primera es la de sujeción de elementos físicos que realizan la alimentación, guía y pelado; y, la segunda, es la de separación de productos, esto es, el direccionamiento de los granos de maíz que puedan desgranarse hacia un punto y la hoja hacia otro.

El sistema de soporte se desarrolló con un perfil angular de acero A36. Para realizar la separación de los granos que se desgranan (condición no deseada en este caso) se utilizó una malla de alambre de acero galvanizado, con orificios cuadrados de $25,4 \mathrm{~mm}$ de lado colocada bajo los rodillos con una inclinación de entre $25^{\circ}$ y $35^{\circ}$.

\section{SISTEMA ELÉCTRICO}

El sistema eléctrico comprende el grupo de componentes que permiten al motor DC proporcionar la potencia necesaria para realizar el proceso.

Para determinar la fuerza de rozamiento y la fuerza total para arrancar la envoltura del maíz se realizaron una serie de pruebas de laboratorio que permitieron determinar estos parámetros.

La fuerza de arranque de la hoja de maíz se determinó utilizando un equipo de ensayos universales de $5000 \mathrm{~N}$. El gráfico 6 muestra los ensayos realizados para determinar la fuerza requerida en el arranque de la hoja de maíz.

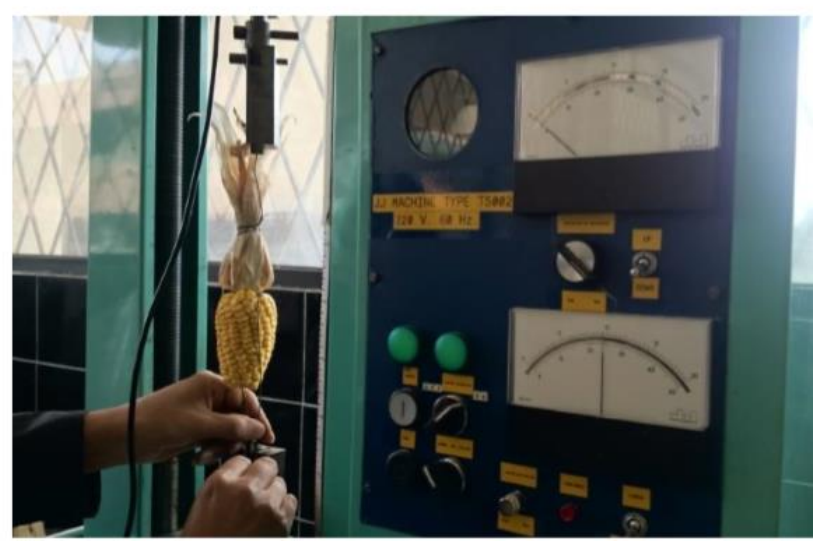

Gráfico 6. Ensayos de fuerza para arranque de la hoja de maíz.

Fuente: elaboración propia. 
La fuerza máxima obtenida para arrancar la envoltura de maíz fue de $125 \mathrm{~N}$.

Para determinar la fuerza de rozamiento se utilizó un plano inclinado que nos permitió determinar el ángulo de deslizamiento del caucho sobre la hoja de maíz.

La fuerza de rozamiento depende del tamaño y peso de la mazorca. La variedad estudiada corresponde al tipo II establecida en la NORMA TÉCNICA ECUATORIANA INEN 1761, cuyos valores están entre $4 \mathrm{~cm}$. y $6,9 \mathrm{~cm}$. de diámetro. El peso promedio de las mazorcas determinado de manera experimental fue de $0,145 \mathrm{Kg}$.

Con esta información se determinó que el torque requerido para el proceso de una mazorca es de 5,3 $\mathrm{N}-\mathrm{m}$.

La potencia del motor depende de la cantidad de producto que se requiera procesar y, por tanto, de la capacidad de producción del equipo. Con los datos obtenidos se determinó que utilizando un motor de 0,373 Kw. (0,5 HP) se podrían procesar al menos 5 mazorcas en el mismo instante de tiempo.

\section{PRUEBAS Y REFINAMIENTO}

Las pruebas y determinación de parámetros adecuados de trabajo se realizaron mediante el método de diseño de experimentos. Se identificaron los parámetros de control y los de ruido. Se determinó como parámetros de control a los siguientes:

- Velocidad de rotación de los rodillos

- Inclinación de los rodillos

- Material de los rodillos

- Acabado superficial de los rodillos

- Humedad relativa de la mazorca de maíz.

Como único parámetro de ruido se consideró:

- Vibración del equipo

Una vez definidos estos parámetros, asumiendo una incidencia mínima de la vibración, se aplicó una matriz de trabajo tipo $L 8$ a 2 niveles, sin influencia de ruidos.

A continuación, se muestra la matriz utilizada y los resultados obtenidos: 


\begin{tabular}{|c|c|c|c|c|c|c|}
\hline Prueba No. & $\begin{array}{c}\text { Velocidad de } \\
\text { rotación } \\
\text { (RPM) }\end{array}$ & $\begin{array}{c}\text { Inclinación de } \\
\text { los rodillos } \\
\text { (o) }\end{array}$ & $\begin{array}{c}\text { dureza del } \\
\text { material } \\
\text { (Shore A) }\end{array}$ & $\begin{array}{c}\text { Acabado } \\
\text { superficial }\end{array}$ & $\begin{array}{c}\text { Humedad } \\
\text { Relativa } \\
\text { (\%) }\end{array}$ & $\begin{array}{c}\text { Resultado } \\
\text { (\% producto } \\
\text { conforme) }\end{array}$ \\
\hline 1 & 600 & 5 & 30 & liso & 15 & 87 \\
\hline 2 & 600 & 5 & 30 & rugoso & 10 & 90 \\
\hline 3 & 600 & 10 & 75 & liso & 15 & 95 \\
\hline 4 & 600 & 10 & 75 & rugoso & 10 & 96 \\
\hline 5 & 300 & 5 & 75 & liso & 10 & 92 \\
\hline 6 & 300 & 5 & 75 & rugoso & 15 & 98 \\
\hline 7 & 300 & 10 & 30 & liso & 10 & 90 \\
\hline 8 & 300 & 10 & 30 & rugoso & 15 & 99 \\
\hline
\end{tabular}

Tabla 1. Resultados de una matriz de trabajo tipo L8 a 2 niveles.

Fuente: elaboración propia.

La mejor combinación en las pruebas desarrolladas se encontró con humedad relativa de la mazorca de maíz de 15\%, material del rodillo con rugosidad y una dureza del mismo en un valor de 75 shore A. La inclinación y velocidad podrían estar en cualquiera de los niveles.

\section{CONCLUSIONES}

a) El diseño de los rodillos permite disminuir la longitud de los mismos, respecto a los diseños encontrados tanto en patentes como en los que se encuentran en el mercado, lo cual hace de este desarrollo una opción de menor costo.

b) Debido a las dimensiones y operación del prototipo, un motor de $1 / 2 \mathrm{HP}$ logra obtener resultados satisfactorios en el trabajo.

c) Los equipos similares encontrados en el mercado, debido a su diseño y características particulares, no logran realizar el trabajo para la variedad de producto en estudio, pues un alto porcentaje de grano es dañado al procesar la mazorca en máquinas comerciales.

d) La utilización de dos rodillos es suficiente para la realización del trabajo en esta variedad de maíz.

e) La utilización de un distinto acabado superficial en los rodillos, respecto a los encontrados comercialmente, es posible en el diseño de los rodillos. Las pruebas realizadas demostraron que incluso los rodillos lisos generan un rozamiento capaz de efectuar el proceso aunque presentan menor eficiencia.

f) La utilización de cauchos de menor dureza presentan desgastes en la superficie y contaminación del producto.

g) Los resultados obtenidos relativos a formas, tamaños, dureza de materiales, potencias, son exclusivamente para la variedad estudiada (maíz seco suave de altura); en caso de aplicaciones a otras variedades, podría necesitarse un ajuste de parámetros de trabajo. 
h) Se comprobó la eficacia de trabajo con el diseño desarrollado que es en promedio del $98 \%$, específicamente en la medición de efectividad en el arranque de la envoltura.

i) El diseño desarrollado disminuye el costo del equipo en aproximadamente un $75 \%$ del precio de equipos similares existentes en el mercado.

j) La modularidad del diseño presentado permite realizar cambios y/o adiciones de acuerdo a la necesidad, por ello este equipo podría utilizarse para distintas variedades de maíz, para otros productos e incluso para distintas aplicaciones como trilla.

k) La capacidad del equipo puede variar dependiendo de la potencia del motor que se utilice.

I) La longitud de los rodillos no influye en la capacidad del equipo. 


\section{REFERENCIAS BIBLIOGRÁFICAS}

CEPAL, FAO, IICA. (2014). Perspectivas de la agricultura y del desarrollo rural en las américas: una mirada hacia América Latina y el Caribe . San José C.R.: IICA.

Cruz, P., Romantchick, E., Hahn, F. (2010). Diseño, construcción y evaluación de una máqquina pra deshojar la mazorca de maíz para la envoltura de tamal. Ingeniería agrícola y Biosistemas, 69 $-74$.

Cruz, P., Romantchick, E., Hahn, F. (2010). Diseño, construcción y evaluación de una máquina para deshojar la mazorca de maíz para envoltura de tamal. Ingeniería Agrícola y Biosistemas, 2(2), 69-74.

De los Ríos, J. (2008). La agricultura latinoamericana y sus posibilidades de adapatación a los cambios climáticos globales. IDeAS, 2(1), 53-75.

Donoso, J. (2007). Situación del sector de maquinaria agrícola en América Latina. Rosario: STRAT Consulting.

Enac, J. y Enac L. (1970.). España. Patente no 384194.

Inurritegui, M., Camacho, I., Ludeña, J. (2008). Acuerdo Asociación UE-CAN: ¿Quienes ganan y quienes pierden? Un estudio exploratorio sobre el sector agrícola. Lima: Centro Peruano de Estudios Sociales.

Moya, C. (2014). Diseño y construcción de una máquina deshojadora de maíz seco que partindo de la mazorca con su envoltura, entregará como producto la mazorca y como subproducto, la envoltura del maíz. Sangolquí.

Ulrich, K., Eppinger, S. (2013). Diseño y desarrollo de productos (Quinta ed.). México: Alfaomega. 